\title{
Tmoato industrial derivatives: mallardo reaction and residual allergenicity
}

\author{
Valerio Pravettoni ${ }^{*}$, Laura Primavesi ${ }^{1}$, Marta Piantanida ${ }^{1}$, Oreste V Brenna ${ }^{2}$, Larua Farioli ${ }^{3}$, Joseph Scibilia ${ }^{4}$, \\ Ambra Mascheri ${ }^{4}$, Elide Anna Pastorello ${ }^{4}$ \\ From Food Allergy and Anaphylaxis Meeting 2011 \\ Venice, Italy. 17-19 February 2011
}

\section{Background}

tomato is the third most cultivated crop around the world, $4.64 \%$ of which derived from Italy, where tomato production is mainly focused on industrial derivatives (about $85 \%$ ).In a previous study we demonstrated that, differently from other lipid transfer protein (LTP) containing fruits, in tomatoes LTP is contained in peel, pulp and seeds.

\section{Aim of the study}

To investigate the influence of technological processing, usually applied in tomato industry, on the allergenicity of the end product in respect to fresh tomato fruit. We analyzed twenty-two processed tomato derivatives purchased on Italian market, and two tomato extracts obtained from whole tomatoes and chemically peeled tomatoes.

\section{Methods}

The thermal damage index in all tomato derivatives was determined chromatographically by detecting furosine level, which allowed us to divide the commercial products in low, medium and highly thermally damaged. SDS-PAGE and immunoblotting on samples of these three groups was performed. We used the patients' sera from our previous study after obtaining informed consent. Five patients had a documented positive history of severe allergic reactions to tomato, fresh or household cooked or industrially processed, and were exclusively reacting to tomato LTP. Other five patients experienced oral allergy syndrome (OAS) grade I-II when eating fresh tomatoes and were sufferings from birch pollinosis and not reacting to tomato LTP.

\footnotetext{
'Foundation IRCCS Cà Granda Ospedale Maggiore Policlinico, Clinical Allergy and Immunology Unit, Milan, Italy

Full list of author information is available at the end of the article
}

\section{Results}

In LTP-positive patients, no statistical difference between chemically peeled and raw extracts was detected by means of skin tests. Any grade of thermal damage (low, medium or high furosine index) induced a significant reduction in tomato allergenicity in birch pollen-positive LTP-negative patients, while none of the investigated technological processes reduced the IgEbinding to tomato LTP in LTP-positive patients.

\section{Conclusions}

LTP-positive patients with clinical symptoms to tomatoes should avoid commercial tomato products even if subjected to high thermal treatment.

\section{Author details \\ ${ }^{1}$ Foundation IRCCS Cà Granda Ospedale Maggiore Policlinico, Clinical Allergy and Immunology Unit, Milan, Italy. ${ }^{2}$ University of Mllan, Dipartimento di Scienze e Tecnologie Alimentari e Microbiologiche (DISTAM), Milan, Italy. ${ }^{3}$ Niguarda Cà Granda Hospital, Laboratory Medicine, Milan, Italy. ${ }^{4}$ Niguarda Cà Granda Hospital, Allergology and Immunology, Milan, Italy.}

Published: 12 August 2011

doi:10.1186/2045-7022-1-S1-P19

Cite this article as: Pravettoni et al:: Tmoato industrial derivatives: mallardo reaction and residual allergenicity. Clinical and Translational Allergy 2011 1(Suppl 1):P19.

\section{Biomed Central}

(c) 2011 Pravettoni et al; licensee BioMed Central Ltd. This is an open access article distributed under the terms of the Creative Commons Attribution License (http://creativecommons.org/licenses/by/2.0), which permits unrestricted use, distribution, and reproduction in any medium, provided the original work is properly cited. 